\title{
Estudio de los hábitos de desplazamiento del jabalí (Sus scrofa Linnaeus, 1758) mediante técnicas gps/gsm en un área de montaña mediterránea
}

\section{INTRODUCCIÓN $^{1}$}

La tecnología GPS permite la captura en tiempo real de numerosos datos relacionados con el posicionamiento geográfico de las especies, superando en cantidad y calidad la información proporcionada por los antiguos sistemas de seguimiento. Por otra parte, las redes de telefonía móvil (GSM) han favorecido el desarrollo de sistemas híbridos que permiten enviar la información capturada en campo al gabinete. Los modelos resultantes, de la aplicación de estas técnicas de seguimiento en campo, son eficaces para establecer planes de conservación, buscar nuevas localizaciones de especies raras y amenazadas, determinar los patrones espaciales de distribución de la biodiversidad, o evaluar el impacto del cambio global sobre la distribución de los organismos ${ }^{2}$.

Los vertebrados terrestres se encuentran ligados a las fluctuaciones del medio. La mayoría de las áreas susceptibles de gestión y conservación, general-

${ }^{1}$ Los autores de este proyecto queremos agradecer especialmente a la Fundación C.V. Victoria Laporta Carbonell por las facilidades prestadas para llevar a cabo la investigación en los terrenos de la finca Buixcarró. Del mismo modo, queremos agradecer a la clínica veterinaria «El Terrer» los servicios prestados. Finalmente, se debe mencionar que este proyecto ha sido realizado bajo el marco del Departamento de Ecología y el Instituto de Investigación Multidisciplinar Ramón Margalef de la Universidad de Alicante. Ha financiado en parte por el proyecto de investigación de la Conselleria de Educación y Ciencia GVA-Pre-2008-036 y el Instituto Alicantino de Cultura Juan Gil-Albert.

${ }^{2}$ Arques Pina, José; Belda Antolí, Antonio; Martínez pérez, José Emilio; Peiró Clavell, Victoriano; Jiménez García, Daniel y Seva Román, Eduardo (2009): "Analysis of surveys as a tool sustainable management of hunting species in the East agrosistemas the province of Alicante (Marina Baja): the wild boar case study (Sus scrofa Linnaeus 1758)". Galemys, 1/21, pp. 51-62. 
mente, sufren algún tipo de explotación que se traduce en impactos directos o indirectos ${ }^{3}$. A pesar de esto, pocos trabajos se relacionan directamente con la gestión sostenible de los recursos de interés cinegético ${ }^{4}$.

Con este proyecto se pretende poner de manifiesto la importancia de la conservación de los espacios agroforestales tradicionales, en ambiente mediterráneo, buscando la compatibilidad de los usos del suelo con nuevas economías regionales ligadas a la cultura cinegética. Así, la conservación del entorno natural, en una matriz antropizada -manteniendo un cierto grado de explotación, y, por tanto, la adecuada gestión de las poblaciones de fauna- supone alcanzar una elevada calidad del hábitat y una estructura paisajística compleja con altas tasas de biodiversidad ${ }^{5}$.

\section{ZONA DE ESTUDIO}

El estudio se ha llevado a cabo en la finca Buixcarró, gestionada por la Fundación de la Comunidad Valenciana Victoria Laporta Carbonell (FVLC), situada entre los términos municipales de Alcoi y Bocairente, en el sector suroeste del Parque Natural de la Sierra de Mariola, a caballo entre las provincias de Alicante y Valencia (UTM 702000N-4288000E).

El clima es típicamente mediterráneo con temperaturas suaves, lluvias concentradas en primavera y otoño; y un destacado periodo seco en verano. La vegetación climácica del Termotipo Mesomediterráneo y Ombrotipo subhúmedo es el carrascal (asociación Hedero helicis-Quercetum rotundifoliae subas. ulicerosum parvifl orae). Incluye formaciones arbóreas dominantes de pino carrasco y encina con estructura arbustiva y ocasionalmente arbórea.

\section{Metodología}

El presente estudio se llevó a cabo entre noviembre de 2009 y enero de 2010. Para el ensayo se utilizó un collar de localización GPS/GSM, adaptán-

\footnotetext{
${ }^{3}$ Sanderson, Erik; Redford, Kent; Vedder, Amy; Coppolillo, Peter \& Ward, Sarah (2002): "A conceptual model for conservation planning based on landscape species requirements". Landscape and Urban Planning, 1/58, pp. 41-56.

${ }^{4}$ Lombardi, Ludgarda; Fernández, Néstor; Moreno, Sacramento \& Villafuerte, Rafael (2003): "Habitat-related differences in rabbit (Oryctolagus cuniculus) abundance, distribution and activity". Journal of Mammalogy, 1/84, pp. 26-36.

${ }^{5}$ Wilkie, Karen; Tyler, Michael \& Alexander, Susann (2003): "Conserving habitat and biodiversity in urbanizing landscapes”. Environmental Informatics Archives, 1/1, pp. 190-196. 
dose a una hembra joven de jabalí. Este dispositivo proporciona datos de los patrones de movimiento y permite determinar distintos aspectos etológicos sobre preferencia de hábitat, capacidad de desplazamiento e interacción con otras especies. Gracias a la información obtenida, enviada mediante SMS e introducida en un sistema de información geográfica (SIG), es posible desarrollar modelos de distribución y comportamiento de la fauna, lo que facilita la implantación de estrategias de gestión y conservación más adecuadas a la realidad ecológica de la especie.

\section{RESULTADOS}

Se ha obtenido una tabla de datos con un total de 4.675 posiciones, durante los 40 días efectivos de muestreo. La distancia total recorrida por esta hembra joven, durante el período de estudio, ha sido aproximadamente de 309 kilómetros y el área de campeo de 44 ha (véase ilustración 1).

\section{ILUSTRACIÓN 1}

\section{MOVIMIENTOS DEL JABALÍ REGISTRADOS MEDIANTE EL COLLAR GPS}

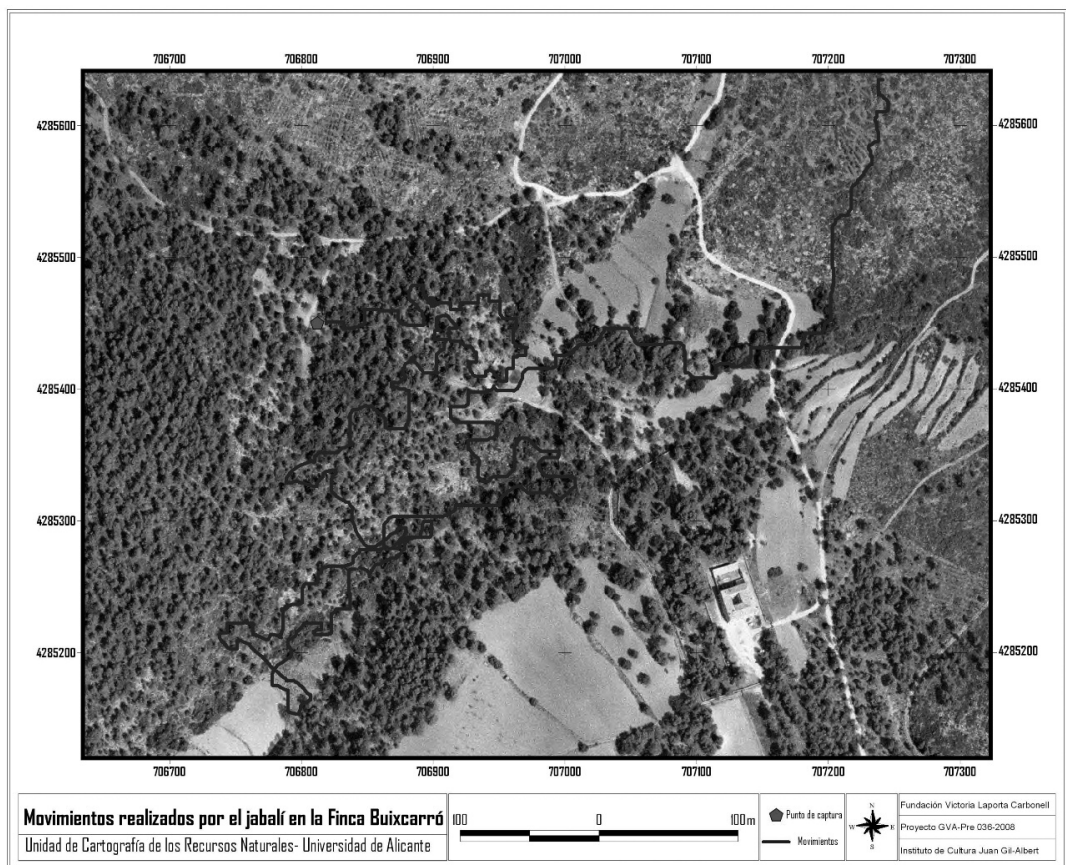

Estudios Geográficos, Vol. LXXVI, 278, pp. 409-416, enero-junio 2015 ISSN: 0014-1496, eISSN: 1988-8546, doi: 10.3989/estgeogr.201515 
La tecnología utilizada en el seguimiento de las especies de fauna permite obtener gran cantidad de información sobre el patrón de uso del espacio por parte de los animales, lo cual contribuye a comprender la dinámica de utilización del espacio ${ }^{6}$. Así, tras analizar los registros de posiciones se ha determinado que el 84,69\% de las posiciones se corresponden con la franja horaria 19:00 a 7:00 horas, resultando muy poca actividad durante las horas centrales del día (véase ilustración 2).

Al analizar el patrón de distribución espacial de la vegetación, en la zona de campeo identificada, se observa una importante heterogeneidad estructural de la misma. Aunque la presencia de áreas de cultivo representa, aproximadamente, entre un 25 y un 30 por ciento del área de estudio, su actividad en relación con ellas, se centra en la zona de contacto con las diferentes formaciones boscosas. No se observa el acceso hacia las plantaciones en hilera de árboles frutales de secano que aparecen entre bancales.

En el patrón de desplazamiento observado se confirma la tendencia aparente, en la preferencia de uso por el jabalí, de las áreas con mayor estructuración de la vegetación y de mayor abundancia de quercíneas y su vegetación asociada. Estos patrones parecen responder, en un análisis ambiental preliminar de la zona, con enclaves cuya ubicación topográfica próxima a vaguadas y con mayor profundidad de suelos asegura una mayor productividad primaria natural (véase tabla 1).

\section{DisCUSIÓN Y CONCLUSIONES}

El dispositivo de localización GPS ha permitido establecer de forma preliminar los principales hábitos de desplazamiento de una especie ampliamente distribuida por la zona de estudio. Este tipo de técnicas permiten la caracterización del patrón de uso del espacio por parte de la fauna. El área de campeo, que viene definida por la disponibilidad de los requerimientos esenciales para una determinada especie, puede calcularse a través de diferentes métodos entre el que destaca la localización GPS. La superficie, la estructura y la forma del área de campeo, en combinación con los elementos del medio físico y biótico, nos proporciona una valiosa información relacionada con la estructura del paisaje ecológico y la relación que establece la especie con el mismo. Así, una de las principales aplicaciones de la localización GPS es establecer las relaciones entre la fauna y los recursos de su entorno. El hábitat proporciona alimento,

\footnotetext{
${ }^{6}$ Rodgers, Arthur (1991): "Recent telemetry technology", in Joshua Millspaugh \& John Marzluff (ed.): Radio tracking and animal populations. California, Academic Press, pp. 79-121. 
TABLA 1

PORCENTAJE DE USO DEL SUELO POR PARTE DEL JABALÍ

\begin{tabular}{lc}
\hline \multicolumn{1}{c}{ Uso del suelo } & Porcentaje de uso \\
\hline Encinar & 19,54 \\
Pinar & 28.52 \\
Matorral arbolado & 12,73 \\
Matorral & 7,84 \\
Vegetación riparia & 16,55 \\
Sembrados & 11,43 \\
Viales & 3,39 \\
\hline
\end{tabular}

refugio, agua y otros recursos esenciales para la supervivencia. El análisis de las relaciones entre el hábitat y el animal poseen una importante componente de gestión de fauna, ya que el estudio de la selección de recursos proporciona información interesante acerca de la importancia relativa del hábitat ${ }^{7}$.

Efectivamente, se ha comprobado que tiene movimientos fundamentalmente crepusculares y una elevada superficie de campeo, coincidiendo con otras regiones mediterráneas ${ }^{8}$. La incorporación de las diferentes localizaciones en el SIG ha permitido, de una manera sencilla, reflejar la información recopilada durante el período de estudio y así poder comprender los hábitos y preferencias de esta especie. De este modo, se pueden determinar los corredores ecológicos y minimizar el impacto que tienen algunas infraestructuras sobre la especie, permitiendo al mismo tiempo reducir el riesgo de colisión con los vehículos ${ }^{9}$.

Se dudaba de la hermeticidad del collar GPS en caso de lluvia e inmersión en agua y lodo, pero ha superado satisfactoriamente estas condiciones, dado que durante este período se han producido fuertes precipitaciones, incluso en forma de nieve. Resulta interesante también destacar que el collar no ha provocado ningún daño en el cuello del animal capturado, tal y como se pudo comprobar al retirarlo del mismo. No obstante, hay que tener en cuenta que el sistema GPS requiere una considerable fuente de energía, lo cual limita el tiempo de operación con este tipo de aparatos, además de suponer un incremento considerable en el peso total del dispositivo. Este aspecto debería con-

\footnotetext{
${ }^{7}$ Belda Antolí, Antonio; Martínez Pérez, José Emilio; Zaragozí Zaragozí, Benito; Ferri Vila, Vicente y Peiró Clavell, Victoriano (2012): "Estimación del hábitat del jabalí (Sus scrofa Linna- 
siderarse a la hora de diseñar este tipo de dispositivos, con el fin de reducir al máximo el peso y volumen de los mismos.

En un estudio en una zona montañosa en los Apeninos (Italia), a tres escalas espaciales diferentes en un rango de parcelas de muestreo entre 25 ha y 5 metros cuadrados, concluyen que el uso del hábitat por jabalí está relacionado con la alimentación y con la protección ante predadores. Indicando la preferencia por vegetación boscosa y madura tanto en bosques caducifolios como mixtos e incluso en bosques de rebrote ${ }^{10}$.

La dieta de jabalí presenta una mayor proporción de alimentos vegetales y una parte importante de estos procede de frutos secos (bellotas, piñones, castañas) y también olivas ${ }^{11}$. Por lo general, estos son frutos invernales mientras que los almendros presentes en la zona presentarían una disponibilidad mayor a principios del otoño. Estos autores reflejan también la importancia de raíces y cereales que representan casi el doble de la dieta de los frutos mencionados en los estudios relativos a España. Los hongos aparecen como un alimento que puede ser significativo aunque dado que el otoño de 2009 ha sido especialmente frío, no ha favorecido la abundancia de este recurso.

En otras formaciones mediterráneas, dominadas por especies del género Pinus, se analizó la actividad del jabalí en el Parque de la Maremma en una zona mediterránea subhúmeda italiana con una vegetación mixta de Pinus pinea, P. pinaster y Quercus ilex. La hipótesis analizada era si el tamaño del área de campeo (home-range) estaba inversamente relacionada con la disponibilidad de alimento, y en particular durante el invierno. No se corroboró la hipótesis, incluso la tendencia en la reducción del home-range coincidió con un periodo de escasez de alimento. Resultó difícil separar el efecto cruzado entre la densidad de población de jabalí y la disponibilidad de alimento. No obstante, hay que destacar que su cuantificación de la disponibilidad de alimento en verano se refirió principalmente a la cantidad de bellota, por lo que un

eus 1758) en el Parque Natural de la Serra de Mariola mediante Sistemas de Información Geográfica". Mediterranea, 1/23, pp. 10-30.

8 Baubet, Eric; Brandt, Serge; Vassant, Jacques; Gendner, Jean \& Klein, Fraçois (2004): "Can wild boar be surveyed using GPS?" National Institute of Polar Research, Special Issue/58, pp. 188-195.

9 Rico Guzmán, Encarnación; Cantó, José; Terrones Contreras, Beatriz \& Bonet Jornet, Andreu (2011): "Impacto del tráfico rodado en el P. N. del Carrascal de la Font Roja. ¿Cómo influyen las características de la carretera en los atropellos de vertebrados?" Galemys, 2/23, 113-123.

${ }^{10}$ Meriggi, Alberto \& Sacchi, Omar (2000): "Habitat requirements of wild boars in the northern Appennines (N Italy): a multi-level approach". Italian Journal of Zoology, 68, pp. 47-55.

11 Schley, Laurent \& Roper, Timothy (2003): "Diet of wild boar Sus scrofa in Western Europe, with particular reference to consumption of agricultural crops". Mammal Review, 1/33, pp. 43-56. 
cambio en el tipo de recurso (raíces, etc.) pudo no ser considerado ${ }^{12}$. Aunque en un estudio previo en la misma zona parece justificar un patrón claro en la repartición estacional del recurso alimenticio utilizado ${ }^{13}$.

En un análisis del patrón de búsqueda de raíces (rooting), analizando esta especie en Suecia, concluye que se observan diferencias significativas entre años, periodo estacional, tipo de hábitat y tipo de suelo. Y que la cantidad de superficie afectada por «rooting» variaba entre 2,4 y 14,2 hectáreas de las 226 ha censadas. Es decir entre un 1,06 y un 6,28 por ciento de la superficie disponible. Las formaciones vegetales eran afectadas en orden decreciente: primero las caducifolias, seguidas de bosques de coníferas y por último los herbazales, destacando en superficie la afectada en suelos húmedos sobre los $\operatorname{secos}^{14}$.

En la Isla de Santa Catalina (California) donde el jabalí fue introducido por los españoles en el siglo XVI (Baber y Coblentz, 1986), cuantificaron la densidad entre 21 y 34 individuos por kilómetro cuadrado. Coinciden también en la reducción del home-range en las épocas secas pero por razones de disponibilidad de agua y ambientes frescos y asocian la disponibilidad de alimento al factor determinante durante las estaciones más húmedas.

En las llanuras costeras de Carolina del Sur, en plantaciones y bosques mixtos dominados por Pinus taeda, Wood y Brenneman (1980) observan una tendencia a permanecer más tiempo en las zonas pantanosas. Los bosques de pino eran utilizados como zonas de descanso y las formaciones de quercíneas eran poco utilizadas, a excepción de los periodos en los que las bellotas estaban disponibles. Tras más de un año de seguimiento estos autores cuantificaban el tamaño del área de campeo media (average home-range) entre 181 y 226 hectáreas y el área de campeo diaria (diel home range) entre 15 y 16,2 hectáreas.

En el patrón de desplazamiento, observado en nuestra zona, se confirma la tendencia aparente hacia las zonas de mayor estructuración en la vegetación y con abundancia de quercíneas y de las especies asociadas a éstas. Parece conveniente proceder a un análisis de los patrones espaciales a escala de paisaje

12 Massei, Giovanna; Genov, Peter; Staines, Brian \& Gorman, Mary (1997): "Factors influencing home range and activity of wild boar (Sus scrofa) in a Mediterranean coastal area". Journal of Zoology, 3/ 242, pp. 411-423.

13 Massei, Giovanna; Genov, Peter \& Staines, Brian (1996): "Diet, food availability and reproduction of wild boar in a Mediterranean coastal area". Acta Theriologica, 3/41, pp. 307-320.

14 Welander, Jonas (2000): "Spatial and temporal dynamics of wild boar (Sus scrofa) rooting in a mosaic landscape". Journal of Zoology, 2/252, pp. 263-271. 
de la riqueza y diversidad de las especies vegetales y hongos que pueden resultar más palatables para el jabalí. Los órganos (raíces, frutos secos, rizomas y setas) identificados como prioritarios deben ser estudiados en su fenología y su evolución interanual.

En un futuro, se pretende colocar este mismo dispositivo sobre otros individuos de esta especie, con el objetivo de ampliar la temática de estudio a diferentes clases de sexo y edad de la población de jabalíes. Además también sería conveniente la ampliación a otras especies de caza mayor, teniendo especial interés el muflón (Ovis musimon), debido a la elevada población que presenta la especie en la zona de estudio. Se asume que los resultados obtenidos serán de elevado interés para otros grupos de investigación, organismos públicos, federaciones de caza, empresas y otras entidades, ya que se podrán minimizar los daños agrícolas y medioambientales que ocasiona esta especie y se podrá optimizar como recurso cinegético, gracias al conocimiento de la utilización de los diferentes usos del suelo.

Antonio Belda, J. E. Martínez-Pérez, V. Ferri y B. Zaragozí Universidad de Alicante 